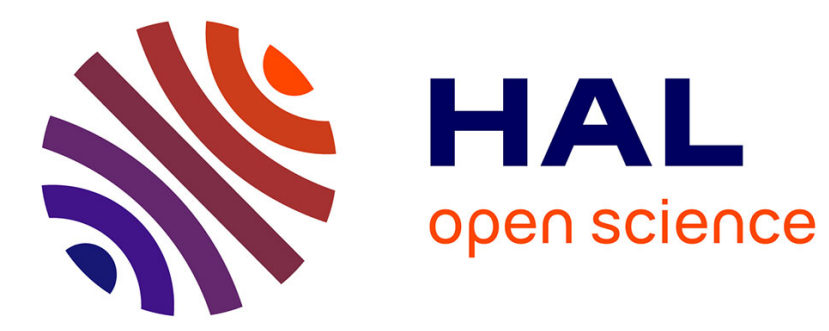

\title{
Near-Optimal Low-Complexity Harmonic Receiver for Unipolar-FSK
}

A. W. Azim, M. J. Khan, O. de Wulf, Yannis Le Guennec, G. Maury, Laurent Ros

\section{- To cite this version:}

A. W. Azim, M. J. Khan, O. de Wulf, Yannis Le Guennec, G. Maury, et al.. Near-Optimal LowComplexity Harmonic Receiver for Unipolar-FSK. IEEE Wireless Communications Letters, 2021, 10 (11), pp.2421-2425. 10.1109/LWC.2021.3102123 . hal-03324540

\section{HAL Id: hal-03324540 https://hal.science/hal-03324540}

Submitted on 23 Aug 2021

HAL is a multi-disciplinary open access archive for the deposit and dissemination of scientific research documents, whether they are published or not. The documents may come from teaching and research institutions in France or abroad, or from public or private research centers.
L'archive ouverte pluridisciplinaire HAL, est destinée au dépôt et à la diffusion de documents scientifiques de niveau recherche, publiés ou non, émanant des établissements d'enseignement et de recherche français ou étrangers, des laboratoires publics ou privés. 


\title{
Near-Optimal Low-Complexity Harmonic Receiver for Unipolar-FSK
}

\author{
A. W. Azim, M. J. Khan, O. De Wulf, Y. Le Guennec, G. Maury, and L. Ros
}

\begin{abstract}
In this letter, we propose a near-optimal lowcomplexity frequency-domain (FD) harmonic receiver for visible light communication based unipolar frequency-shift keying ( $U$ FSK). The time-domain (TD) maximum-likelihood (ML) receiver for U-FSK is computationally complex, while the sub-optimal receiver increases the noise variance. The proposed harmonic receiver determines the transmitted frequency from the crosscorrelations between the FD received waveform and the FD dictionary waveforms reduced to their strongest harmonics. The results shall demonstrate that besides affording a linerarithmic complexity, the performance of the harmonic receiver closely approaches that of TD ML receiver which exhibits quadratic complexity.
\end{abstract}

Index Terms-Discrete cosine transform, intensity-modulation and direct-detection, visible light communications, harmonic receiver, frequency-shift keying.

\section{INTRODUCTION}

$\mathbf{V}$ ISIBLE light communication (VLC) could be a vital integrant for ubiquitous Internet-of-Things to alleviate the burden from radio technologies, in particular addressing energy efficient low data rate communications, using low complexity intensity-modulation and direct-detection (IM-DD) [1]. In our previous work [2], we proposed frequency-shift keying (FSK) based IM-DD compatible modulation schemes, which are; direct-current-FSK (DC-FSK) and unipolar-FSK (U-FSK). The underlying distinction between these approaches exists in the way a non-negative waveform is obtained for IMDD. DC-FSK incorporates a bias, while U-FSK sequentially transmits the positive amplitude excursions in one symbol period and sign flipped negative amplitude excursions in the second symbol period. Though U-FSK is spectrally less efficient than DC-FSK, it yields higher energy efficiency. We refer interested readers to [2], [3], [4] for a detailed description of various FSK schemes and their generalizations for VLC. In this work, we shall restrain our discussion to only U-FSK.

In [2], we proposed time-domain (TD) maximum-likelihood (ML) and sub-optimal receivers for U-FSK. The TD ML receiver determines the transmitted frequency by calculating the TD cross-correlations between the received waveform having $2 M$ chips and each of the possible $M$ transmit waveforms. The sub-optimal receiver, on the other hand, reconstructs the bipolar FSK waveform before computing its discrete cosine transform (DCT). The complexity of TD ML receiver increases quadratically with an increase in alphabet cardinality, $M$, i.e.,

A. W. Azim is with Department of Telecommunication Engineering, University of Engineering and Technology, Taxila, Pakistan (email: aliwaqarazim@gmail.com)

M. J. Khan, O. De Wulf, Y. Le Guennec and L. Ros are with Université Grenoble Alpes, CNRS, Institute of Engineering, Grenoble INP, GIPSALAB, 38000 Grenoble, France (email: \{muhammad-jehangir.khan,yannis.leguennec,laurent.ros,oscar.de-wulf\}@ grenoble-inp.fr).

G. Maury is with Universite Grenoble Alpes, CNRS, Institute of Engineering, Grenoble INP, IMEP-LaHC, 38000 Grenoble, France (email: maury@grenoble-inp.fr).
$M^{2}$ (if we only count multiplications of non-zero elements), whereas, the complexity of sub-optimal receiver increases linearithmically, i.e., $(M / 2) \log _{2}(M)$. This higher complexity of TD ML receiver makes it less practical, notably for higher $M$. Moreover, the reconstruction step for the sub-optimal receiver doubles the noise variance of the reconstructed bipolar waveform culminating in degraded performance. We shall provide a succinct summary of these receiver architectures in the sequel.

In this letter, we propose a near-optimal low-complexity frequency-domain (FD) $L$-tap harmonic receiver for U-FSK. It is recalled that for bipolar FSK, a single harmonic contains all of the energy, while the number of harmonics containing most of the energy is higher for U-FSK because of clipping and sequential transmission. However, the spectral analysis of U-FSK waveform indicates that only a few harmonics contain most of the energy. The proposed $L$-tap harmonic receiver extracts these high energy harmonics off-line and then determines the transmit frequency by evaluating FD cross-correlation between these harmonics for each possible candidate and FD received waveform (for the given symbol period). Since the number of harmonics, $L$, needed for the FD cross-correlation is low compared to $2 M$ chips used in the TD correlation, the complexity of the proposed receiver reduces significantly. The results provided in the sequel shall indicate that the performance of the proposed receiver is very close to that of TD ML receiver in both additive white Gaussian noise (AWGN) and time-dispersive channels.

We organize the rest of the article as follows. Section II presents a concise summary of waveform design, state-of-theart receiver configurations, and theoretical bit-error-rate (BER) for U-FSK. In Section III, we present the proposed $L$-tap harmonic receiver. Section IV compares the performance of the proposed receiver with conventional receivers in terms of complexity, BER in AWGN and time-dispersive channels and energy efficiency versus spectral efficiency performance. Finally, based on simulation results, conclusions are rendered in Section V.

\section{U-FSK FRAMEWORK}

\section{A. U-FSK Waveform Design}

The $m$ th bipolar FSK discrete waveform having $\tilde{M}_{\mathrm{c}}=$ $M$ chips is $\tilde{\boldsymbol{s}}_{m}=\left[\tilde{s}_{m}[0], \tilde{s}_{m}[1], \cdots, \tilde{s}_{m}\left[\tilde{M}_{\mathrm{c}}-1\right]\right]^{\mathrm{T}}$ with $m \in \llbracket 0, M-1 \rrbracket$, where [. $]^{\mathrm{T}}$ indicates transpose. The symbol duration of $\tilde{\boldsymbol{s}}_{m}$ is $\tilde{T}_{\mathrm{s}}=\tilde{M}_{\mathrm{c}} T_{\mathrm{c}}$, where $T_{\mathrm{c}}$ is the chip duration. The bandwidth occupied by $\tilde{s}_{m}$ is $\tilde{B}=M \Delta f$ where $\Delta f$ is the frequency separation between two adjacent waveforms. $\tilde{\boldsymbol{s}}_{m}$ in its discrete form is given as [2]

$$
\tilde{s}_{m}[n]=\left\{\begin{array}{ll}
\frac{A}{\sqrt{2}} & m=0, \\
A \cos \left[\frac{\pi m}{M}\left(n+\frac{1}{2}\right)\right] & 1 \leq m \leq M-1
\end{array},\right.
$$


for $n \in \llbracket 0, \tilde{M}_{\mathrm{c}}-1 \rrbracket$, where $A$ is the waveform amplitude. The $m$ th U-FSK waveform, $\boldsymbol{s}_{m}=$ $\left[s_{m}[0], s_{m}[1], \cdots, s_{m}\left[M_{\mathrm{c}}-1\right]\right]^{\mathrm{T}}$ having $M_{\mathrm{c}}=2 M$ chips is generated using $\tilde{\boldsymbol{s}}_{m}$ as

$s_{m}[\bar{n}]= \begin{cases}\frac{1}{2}\left(\tilde{s}_{m}[\bar{n}]+\left|\tilde{s}_{m}[\bar{n}]\right|\right) & \bar{n} \in \llbracket 0, M_{\mathrm{c}} / 2-1 \rrbracket \\ -\frac{1}{2}\left(\tilde{s}_{m}[\bar{n}-M]-\left|\tilde{s}_{m}[\bar{n}-M]\right|\right) & \bar{n} \in \llbracket M_{\mathrm{c}} / 2, M_{\mathrm{c}}-1 \rrbracket\end{cases}$

For a given $T_{\mathrm{c}}$, the symbol period and bandwidth for $\boldsymbol{s}_{m}$ are $T_{\mathrm{s}}=2 \tilde{T}_{\mathrm{s}}=M_{\mathrm{c}} T_{\mathrm{c}}$ and $B=2 \tilde{B}=2 M \Delta f$, respectively. In [2], we adopted an optimistic approximation that $B=\tilde{B}$, however, the clipping operation expected to construct the UFSK waveform at least doubles the bandwidth relative to bipolar FSK. Moreover, the maximum spectral efficiency of U-FSK signaling is $\eta=\log _{2}(M) / 2 M$. From [2], we likewise gather that the minimum squared Euclidean distance for UFSK waveform in AWGN channel having electrical symbol energy of $E_{\mathrm{s}_{(\text {elec })}}=\left\|\boldsymbol{s}_{m}\right\|^{2}=\left(A^{2} / 2\right) T_{\mathrm{s}}$ is $d_{\text {min }}^{2} \approx 0.55 \times 2 E_{\mathrm{s}_{(\text {elec })}}$, where $\|\cdot\|^{2}$ evaluates Euclidean norm of the argument.

\section{B. Conventional Receiver Architectures}

1) Maximum-Likelihood Receiver: Considering the same $E_{\left.\mathrm{s}_{(\text {elec }}\right)} \forall m$ and a priori equiprobability for the $M$ transmit waveforms, i.e., $p\left(\boldsymbol{s}_{m}\right)=1 / M$, the ML criterion dictates to maximize the conditional probability density of receiving $\boldsymbol{r}=$ $\left[r[0], r[1], \cdots, r\left[M_{\mathrm{c}}-1\right]\right]^{\mathrm{T}}$ when $\boldsymbol{s}_{m}$ is sent, i.e., $p\left(\boldsymbol{r} \mid \boldsymbol{s}_{m}, \boldsymbol{h}\right)$, where $\boldsymbol{h}=[h[0], h[1], \cdots, h[k-1]]^{\mathrm{T}}$ is $k \geq 1$ length impulse response vector which is assumed to be known at the receiver. In the absence of inter-symbol interference (ISI), which is a reasonable assumption if the delay spread $\Delta \tau=(k-1) T_{\mathrm{c}}$ is substantially smaller than the $T_{\mathrm{s}}$, i.e., $\Delta \tau \ll T_{\mathrm{s}}$, then the transmit frequency is identified using ML criterion as

$$
\hat{m}=\underset{m}{\arg \max }\left\{\left\langle\boldsymbol{r}, \boldsymbol{h} \otimes \boldsymbol{s}_{m}\right\rangle-\frac{1}{2}\left\|\boldsymbol{h} \otimes \boldsymbol{s}_{m}\right\|^{2}\right\},
$$

where $\langle\cdot, \cdot\rangle$ and $\otimes$ evaluate the dot product and convolution, respectively. The term $1 / 2\left\|\boldsymbol{h} \otimes \boldsymbol{s}_{m}\right\|^{2}$ in (3) counterbalances any energy variation maybe induced due to $\boldsymbol{h}$.

Remark 1. DCT preserves the geometry (distance, norms and angles, etc.), as any isometric transform. Hence, TD crosscorrelation in (3) is equivalent to FD cross-correlation, which leads to write the ML criterion as

$$
\hat{m}=\underset{m}{\arg \max }\left\{\left\langle\boldsymbol{R}, \boldsymbol{T}_{m}\right\rangle-\frac{1}{2}\left\|\boldsymbol{T}_{m}\right\|^{2}\right\},
$$

where $\boldsymbol{R}=\mathcal{C}_{M_{\mathrm{c}}} \boldsymbol{r}=\left[R[0], R[1], \cdots, R\left[M_{\mathrm{c}}-1\right]\right]^{\mathrm{T}}$ and $\boldsymbol{T}_{m}=\mathcal{C}_{M_{\mathrm{c}}}\left(\boldsymbol{h} \otimes \boldsymbol{s}_{m}\right)=\left[T_{m}[0], T_{m}[1], \cdots, T_{m}\left[M_{\mathrm{c}}-1\right]\right]^{\mathrm{T}}$, with $\mathcal{C}_{M}$ being the $M$-order DCT matrix as defined in [2]. Note that $\boldsymbol{T}_{m}$ can be interpolated in a look-up-table (LUT) at the receiver.

Note that we did not present the FD ML receiver in [2] because of its higher complexity, which shall be elaborated in the subsequent section. Here, it is only presented for the sake of clarity because it leads to the proposed receiver of Section III by simplifying the detection problems (3) and (4).

Remark 2. For AWGN channel, i.e., when $k=1$ and $\Delta \tau=0$, $\left\|\boldsymbol{h} \otimes \boldsymbol{s}_{m}\right\|^{2}=\left\|\boldsymbol{T}_{m}\right\|^{2}=E_{\mathrm{s}_{(\text {elec })}}$ is independent of $m$, therefore, the detection problems (3) and (4) simplify to

$$
\hat{m}=\underset{m}{\arg \max }\left\{\left\langle\boldsymbol{r}, \boldsymbol{s}_{m}\right\rangle\right\},
$$

and

$$
\hat{m}=\underset{m}{\arg \max }\left\{\left\langle\boldsymbol{R}, \boldsymbol{S}_{m}\right\rangle\right\},
$$

respectively, where $\boldsymbol{T}_{m}=\boldsymbol{S}_{m}=\mathcal{C}_{M_{\mathrm{c}}} \boldsymbol{s}_{m}=$ $\left[S_{m}[0], S_{m}[1], \cdots, S_{m}\left[M_{\mathrm{c}}-1\right]\right]^{\mathrm{T}}$.

2) Sub-optimal Receiver: Using $\boldsymbol{r}$, a bipolar waveform $\tilde{\boldsymbol{r}}=$ $[\tilde{r}[0], \tilde{r}[1], \cdots, \tilde{r}[M-1]]$ having $M$ chips is constructed by reversing the process of (2) as $\tilde{r}[n]=r[n]-r[n+M]$, where $n \in \llbracket 0, M-1 \rrbracket$. Afterwards, employing an $M$-order DCT, $\tilde{\boldsymbol{R}}=\mathcal{C}_{M} \tilde{\boldsymbol{r}}=[\tilde{R}[0], \tilde{R}[1], \cdots, \tilde{R}[M-1]]^{\mathrm{T}}$ is attained, from which the frequency index is detected as

$$
\hat{m}=\underset{m}{\arg \max }\left\{|\tilde{\boldsymbol{R}}|^{2}\right\},
$$

where $|\cdot|$ evaluates the absolute value of the argument. Even though the sub-optimal receiver is straightforward, the bipolar waveform reconstruction doubles the noise variance for $\tilde{\boldsymbol{r}}$ for the same $E_{\left.\mathrm{s}_{(\text {elec }}\right)}$.

3) Theoretical BER Probability: [5] provides theoretical BER expressions for ML detection of bipolar FSK in AWGN channel which depends on the waveform minimum squared Euclidean distance. In [2], an approximate minimum squared Euclidean distance, $d_{\min }^{2}$ is evaluated for U-FSK (given in Section II A), from which, all the inter-symbol distances do no deviate more than $4.65 \%$. So, by replacing the minimum squared Euclidean distance of the bipolar FSK by the one evaluated for U-FSK as given in Section II, an approximation for theoretical BER for U-FSK in an AWGN channel can be achieved. So, the approximate probability of detecting an erroneous symbol, i.e., $P_{\mathrm{e}}$ for TD/FD ML U-FSK detectors is obtained by numerically solving

$$
P_{\mathrm{e}} \approx \frac{1}{\sqrt{2 \pi}} \int_{-\infty}^{\infty}\left[1-(1-Q(x))^{M-1}\right] e^{\left[-\frac{\left(x-\sqrt{d_{\min }^{2} / N_{0}}\right)^{2}}{2}\right]} \mathrm{d} x,
$$

where $d_{\min }^{2} \approx 0.55 \times 2 E_{\mathrm{s}_{\text {(elec) }}}$ [2], $N_{0}$ is the mono-lateral noise spectral density, and $Q(\cdot)$ is the Gaussian Q-function [5]. Then, using (7) and incurring to alphabet symmetry [5], the probability of bit-error in AWGN, i.e., $P_{\mathrm{b}}$ is evaluated as

$$
P_{\mathrm{b}} \approx \frac{M}{2(M-1)} P_{\mathrm{e}}
$$

\section{Proposed $L$-tap Harmonic Receiver}

As aforesaid, the purpose of the $L$-tap harmonic receiver is to simplify the detection problem (4). Like for detection problem (4), $\boldsymbol{T}_{m}$ could be easily evaluated by sending a sequence of all $M$ potential waveforms, $\boldsymbol{s}_{m}$ through the channel, $\boldsymbol{h}$, and interpolating the result of the DCT in a LUT, without adding complexity in the receiver. As an example, for $m \in\{1,2,13,14\}$ when $M=16\left(M_{\mathrm{c}}=32\right)$ and considering AWGN channel, $\boldsymbol{s}_{m}$ and $\boldsymbol{T}_{m}=\boldsymbol{S}_{m}$ are depicted in Fig. 1. Our objective for the proposed $L$-tap receiver is to attain BER that does not deviate more than $5 \%$ of that of TD ML receiver and to reduce the complexity by using 


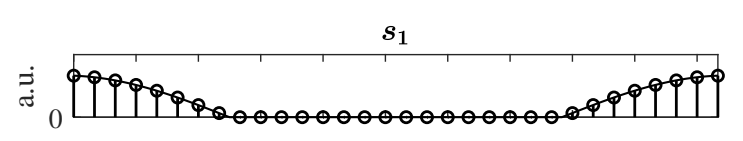

$T_{1}$
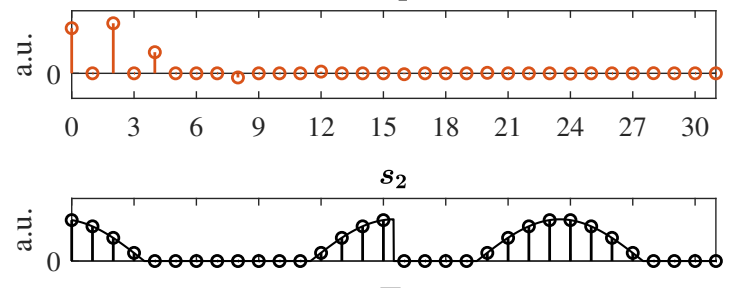

$T_{2}$
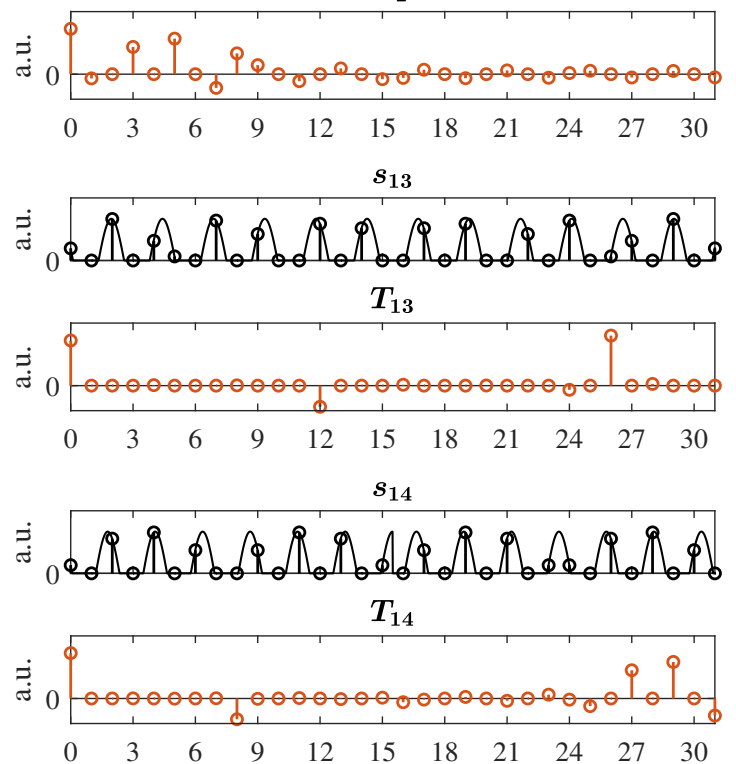

Fig. 1: $\boldsymbol{T}_{m}$ for $m=\{1,2,13,14\}$ considering $M=16$ in an AWGN channel.

a minimum number of harmonics. By analyzing the signal structure using simulation under various system setups, we determine that at least three and thirteen harmonics (including DC harmonic) are required for odd and even frequency waveforms, respectively to fulfill the above-mentioned objectives. Note that this difference in the required number of harmonics is due to the difference in the signal structure that exists for even and odd frequency waveforms (cf. Fig. 1). Furthermore, the energy of these minimum number of harmonics can also be defined in terms of $E_{\mathrm{s}_{(\text {elec }}}$, which is equal to the total energy contained in $\boldsymbol{T}_{\boldsymbol{m}} \forall m$. We determined that we need harmonics containing $90.5 \%$ and at least $97.8 \%$ of $E_{\mathrm{s}_{(\mathrm{elec})}}$ for odd and even frequency waveforms, respectively, irrespective of $M$. For disambiguation, we respectively use $L_{\mathrm{o}}$ and $L_{\mathrm{e}}$ to indicate the number of harmonics for odd and even frequency waveforms. For the proposed receiver, $L_{\mathrm{o}}$ and $L_{\mathrm{e}}$ harmonics of $\boldsymbol{T}_{m}$ are extracted and define the non-zero coefficients of $\boldsymbol{T}_{m}^{L}$, which are interpolated in the LUT. Note that the size of the LUT for the harmonic receiver (defined in terms of number of non-zero coefficients and their indices to store in memory) is significantly less compared to that of the TD ML receiver which would needs a larger LUT.

The receiver architecture of the proposed $L$-tap harmonic receiver is depicted in Fig. 2. The transmit frequency index can be determined by calculating the cross-correlation between

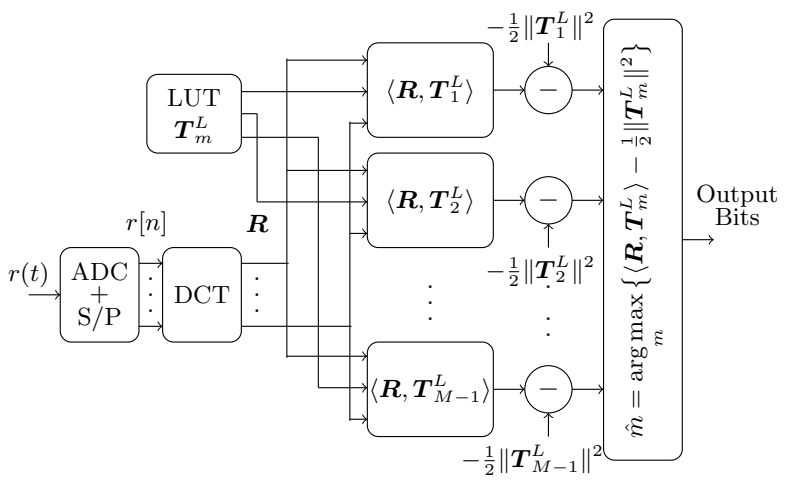

Fig. 2: The architecture of proposed $L$-tap harmonic receiver.

$\boldsymbol{R}$ and $\boldsymbol{T}_{m}^{L}$ and compensating any energy variation that the channel may cause as

$$
\hat{m}=\underset{m}{\arg \max }\left\{\left\langle\boldsymbol{R}, \boldsymbol{T}_{m}^{L}\right\rangle-\frac{1}{2}\left\|\boldsymbol{T}_{m}^{L}\right\|^{2}\right\} .
$$

Since fewer (non-zero) multiplications have to be computed to evaluate the cross-correlation in FD for the proposed $L$-tap receiver, therefore, the complexity of the proposed receiver is substantially lowered compared to the TD/FD ML receivers.

Remark 3. For AWGN channel, owing to the fact that $\boldsymbol{T}_{m}^{L}=$ $\boldsymbol{S}_{m}^{L}=\left[S_{m}^{L}[0], S_{m}^{L}[1], \cdots, S_{m}\left[M_{\mathrm{c}}-1\right]\right]^{\mathrm{T}}$, which represents the FD waveform containing only significant taps defined for AWGN channel, (10) simplifies to

$$
\hat{m}=\underset{m}{\arg \max }\left\{\left\langle\boldsymbol{R}, \boldsymbol{S}_{m}^{L}\right\rangle\right\} .
$$

Remark 4. The DC harmonic which is related to $E_{\mathrm{s}_{(\mathrm{elec})}}$ does not change in AWGN channel, so the DC component is constant for any $\boldsymbol{s}_{m} \forall m \in \llbracket 0, M-1 \rrbracket$, therefore, it can be discarded to keep $L_{\mathrm{o}}-1$ and $L_{\mathrm{e}}-1$ harmonics to evaluate (11). On the other hand, for time-dispersive channel, the symbol energy, $E_{\mathrm{s}_{(\text {elec })}}=\left\|\boldsymbol{h} \otimes \boldsymbol{s}_{m}\right\|^{2}$ is not constant for all $m$, and thus, DC harmonic cannot be discarded to evaluate (10).

\section{Performance Analysis}

In this section, we evaluate and compare the performance of proposed $L$-tap harmonic receiver in terms of complexity, BER in AWGN and time-dispersive channels and energy-efficiency performance for different spectral efficiencies.

\section{A. Complexity Analysis}

The dominant contributor to receiver complexity is the number of multiplications involved for all the operations required from receiving the signal to the determination of the frequency index. Accordingly, the complexity of the proposed receiver and other counterparts is only appraised in terms of required number of multiplications.

The TD ML receiver evaluates the cross-correlation between $M_{\mathrm{c}}$ chips of $\boldsymbol{r}$ and each of the $M$ possible transmit waveforms $s_{m}$, thus, expecting $C_{\mathrm{TD}, \mathrm{ML}}=M M_{\mathrm{c}}$ multiplications. For FD ML receiver, in addition to $C_{\mathrm{TD}, \mathrm{ML}}$ multiplications, $M_{\mathrm{c}^{-}}$ order DCT is needed, therefore, the total number of required multiplications is $C_{\mathrm{FD}, \mathrm{ML}}=\left(M_{\mathrm{c}} / 2\right) \log _{2}\left(M_{\mathrm{c}}\right)+M_{\mathrm{c}} M$; which is higher than that of TD ML counterpart. For the sub-optimal 
TABLE I: $\beta$ for different $M$ considering the AWGN case, i.e. $k=1$ and time-dispersive case, i.e., $k>1$.

\begin{tabular}{c||c||c}
\hline \hline$M$ & $\beta[\%],(k=1)$ & $\beta[\%],(k>1)$ \\
\hline \hline 16 & 62.5 & 59.38 \\
32 & 79.69 & 78.13 \\
64 & 89.06 & 88.28 \\
128 & 94.14 & 93.75 \\
256 & 96.88 & 96.68 \\
512 & 98.34 & 92.24 \\
1024 & 99.12 & 99.07 \\
\hline \hline
\end{tabular}

receiver, only a $M$-order DCT is needed, which requires $C_{\text {sub-opt }}=(M / 2) \log _{2}(M)$ multiplications [6], which is, much less compared to that of the TD/FD ML receivers. Lastly, for the proposed $L$-tap harmonic receiver, besides $\left(M_{\mathrm{c}} / 2\right) \log _{2}\left(M_{\mathrm{c}}\right)$ multiplications needed for $M_{\mathrm{c}}$-order DCT, $(M / 2) L_{\mathrm{o}}$ and $(M / 2) L_{\mathrm{e}}$ multiplications are also needed to evaluate the FD cross-correlation, thus $C_{\text {harm }}=\left(M_{\mathrm{c}} / 2\right) \log _{2}\left(M_{\mathrm{c}}\right)+$ $(M / 2) L_{\mathrm{o}}+(M / 2) L_{\mathrm{e}}$ multiplications are required.

In order to exemplify clearly the complexity reduction of the proposed receiver over TD ML one, we introduce $\beta=$ $\left(1-C_{\text {harm }} / C_{\mathrm{TD}, \mathrm{ML}}\right) \times 100 \%$; which appraises the percentage decrease in complexity of proposed $L$-tap harmonic receiver relative to TD ML receiver. $\beta$ is provided in Table $\mathrm{I}$ for different $M$, for both AWGN $(k=1)$ and time-dispersive $(k>1)$ channels. The slight difference in $\beta$ for AWGN and time-dispersive channel is due to the fact that when we consider time-dispersive scenario, the DC harmonic has to be considered to identify the frequency index, whereas for AWGN channel it is not needed. From Table I, we can gather that the proposed receiver is significantly less complex compared to the TD ML receiver. On the other hand, we may also notice that $C_{\text {sub-opt }}=(M / 2) \log _{2}(M)$ is even less than $C_{\text {harm }}$, however, it shall become clear in the sequel that the performance enhancement in terms of energy efficiency realized using the $L$-tap harmonic receiver over the suboptimal receiver outweighs this limitation.

\section{B. BER Performance Analysis}

In this sub-section, we present and compare the BER performance against signal-to-noise ratio per bit, $E_{\mathrm{b}_{(\text {elec })}} / N_{0}$ where $E_{\mathrm{b}_{(\text {elec })}}=E_{\mathrm{s}_{(\text {elec })}} / \log _{2}(M)$ (evaluated at the receiver input) of the proposed receiver with the state-of-the-art receivers in AWGN and time-dispersive channels. We shall use $\left\{L_{\mathrm{o}}, L_{\mathrm{e}}\right\}=\{2,12\}$ and $\left\{L_{\mathrm{o}}, L_{\mathrm{e}}\right\}=\{3,13\}$ for AWGN and time-dispersive channels, respectively. In the sequel, we use the nomenclature $\left(L_{\mathrm{e}}, L_{\mathrm{o}}\right)$ harmonic receiver for the proposed architecture for clarity. Moreover, even though the performance of FD ML receiver is theoretically strictly equal to that of TD counterpart, we do not provide its performance in the results since its complexity is higher than the one of TD ML receiver (cf. Section IV A).

For BER performance in AWGN channel (cf. Fig. 3), we consider $L_{\mathrm{e}}=\{2,5,12\}, L_{\mathrm{o}}=2$ and $M=16$. Note that different values are considered for $L_{\mathrm{e}}$ in order to highlight the performance improvement while increasing $L_{\mathrm{e}}$. The theoretical BER from (8) and (9) and the BER performances of TD ML and sub-optimal receivers are used as benchmark. It is recalled

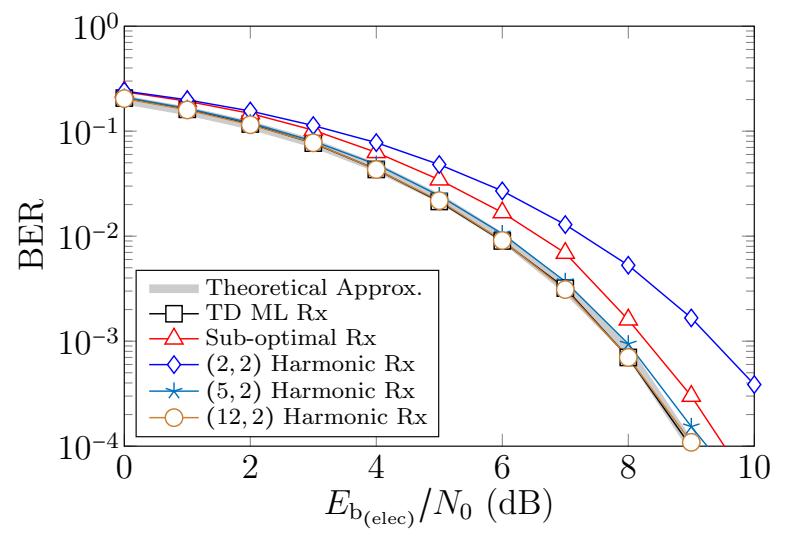

Fig. 3: BER performance against $E_{\mathrm{b}_{(\text {elec })}} / N_{0}$ in AWGN channel considering $M=16$.

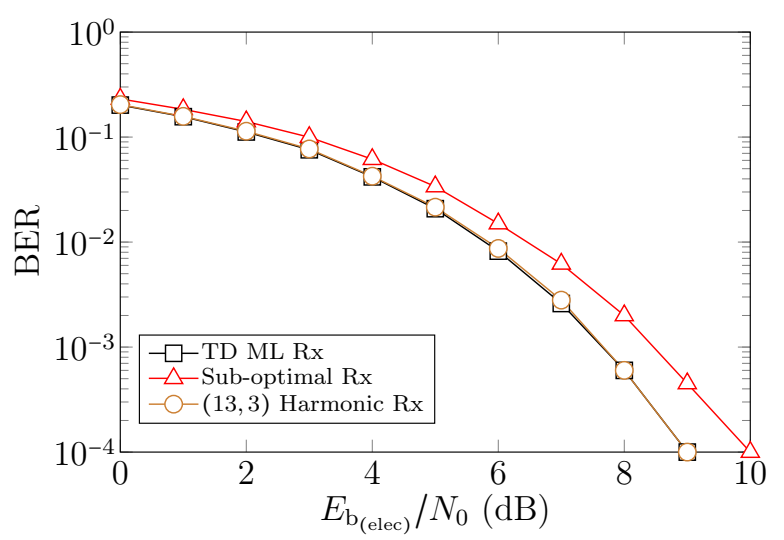

Fig. 4: BER performance against $E_{\mathrm{b}_{(\text {elec })}} / N_{0}$ for time-dispersive channel considering $M=16$.

that (8) and (9) provide the theoretical BER performance for $\mathrm{ML}$ receivers and obtaining analytical results considering $\left(L_{\mathrm{e}}, L_{\mathrm{o}}\right)$ could be a cumbersome task which demands a separate study. From the results depicted in Fig. 3, we note the following: (i) the performance of TD ML receiver is in conformity with the theoretical BER; (ii) the performance of proposed $\left(L_{\mathrm{e}}, L_{\mathrm{o}}\right)$ harmonic receiver improves by increasing $L_{\mathrm{e}}$ and the optimal performance is obtained when $L_{\mathrm{e}}=12$, deviating no more than $5 \%$ relatively to the performance of optimal ML receiver; and (iii) the sub-optimal receiver exhibits approximately $0.6 \mathrm{~dB}$ performance deterioration relative to the counterparts because it doubles the noise variance. Here, $\beta \approx 62.5 \%$ signifies a noteworthy reduction in complexity for the proposed receiver relative to the TD ML receiver for $M=16$.

Fig. 4 illustrates the BER performance of the proposed $\left(L_{\mathrm{e}}, L_{\mathrm{o}}\right)$ harmonic receiver considering $M=16$ in timedispersive channel and compares it with that of TD ML and sub-optimal receivers. The time-dispersive channel is emulated using ceiling bounce model considering data rate of $500 \mathrm{kbps}$ and $\Delta \tau=10 \mathrm{~ns}$ [7]. We only consider $(13,3)$ harmonic receiver. We again observe that the BER performance of proposed $\left(L_{\mathrm{e}}, L_{\mathrm{O}}\right)$ harmonic receiver is very close to that of TD ML receiver with $\beta=59.38 \%$. On the other hand, both harmonic and TD ML receivers achieve significant gain over the sub-optimal receiver because they accommodate the 
channel impulse response into the detection criterion unlike the sub-optimal receiver.

For $\Delta \tau$ of $10 \mathrm{~ns}$, data rate of $500 \mathrm{kbps}$ and $M=16$, we expect a weak ISI since $\Delta \tau \ll T_{\mathrm{s}}$, where $T_{\mathrm{s}}=4 \mu \mathrm{s}$. This ISI-free feature is even more evident for higher $M$ as $T_{\mathrm{s}}$ increases from $4 \mu \mathrm{s}$ for $M=16$ to $10 \mu \mathrm{s}$ for $M=1024$ [2]. On the other hand, for the given data rate, the bandwidth of the U-FSK waveform for $M=16$ is $B=4 \mathrm{MHz}$, which is substantially less than the coherence bandwidth of the channel, $B_{\text {coh }}=1 / \Delta \tau=100 \mathrm{MHz}$. However, since $B$ increases linearly with an increase in $M$, we foresee that the impact of the time-dispersive channel would be more pronounced for higher $M$ because of frequency selectivity of the channel in global U-FSK bandwidth. This impact shall be elaborated in the subsequent section, where we elucidate the energy efficiency performance for different spectral efficiencies.

\section{Energy Efficiency versus Spectral Efficiency}

Fig. 5 and Fig. 6 demonstrate the evolution of required $E_{\mathrm{b}_{(\text {elec }}} / N_{0}$ with respect to $\eta$ for a target BER of $10^{-3}$ in AWGN and time-dispersive channels, respectively. $\eta$ is varied by changing $M$. Fig. 5 signifies that the performance of the proposed $L$-tap receiver is close to that of the TD ML receiver with significant complexity reduction as indicated in Table I. The $E_{\mathrm{b}_{\text {(elec) }}} / N_{0}$ required for the target BER for the proposed receiver is strictly within $5 \%$ of that of TD ML receiver. As expected, the energy efficiency increases with increase in $M$. However, the energy efficiency for the proposed receiver increases more relative to the sub-optimal receiver with an increase in $M$ (cf. Fig. 5).

On the other hand, the energy efficiency versus spectral efficiency results obtained for time-dispersive channel exhibits a different trend for high $M$. We observe that the energy efficiency of the benchmark and the proposed receivers increases up to a certain value of $M$ and then it starts to degrade. The value of $M$ up to which the energy efficiency increases with increase in $M$ is 128 . After that, the condition $B \ll B_{\text {coh }}$ is loosely satisfied due to the increase in $B$ which implies frequency selectivity in the U-FSK bandwidth. Thus, for a given $E_{\mathrm{s}_{(\text {elec })}}, d_{\text {min }}^{2}$ degrades through the channel with increase in $M$ due to strongest distortion of the waveforms. Moreover, because of the frequency selectivity, the correct detection of frequency bins with strong attenuation is poorer which generates errors.

Finally, although the potential of the proposed receiver is apparent, it still has some limitations. For instance, to attain $\boldsymbol{T}_{m}^{L}, \boldsymbol{s}_{m} \forall m \in \llbracket 0, M-1 \rrbracket$ has to be transmitted in advance which increases the latency. Moreover, for detection of transmit frequency, the DC harmonic which may be contaminated by parasitic ambient light [8] has to be used. Therefore, these above-mentioned challenges should be addressed.

\section{CONCLUSION}

In this letter, we propose $L$-tap harmonic receiver which demonstrates near-optimal performance with considerably reduced complexity relatively to TD ML receiver. The proposed receiver simplifies the frequency detection problem by relying

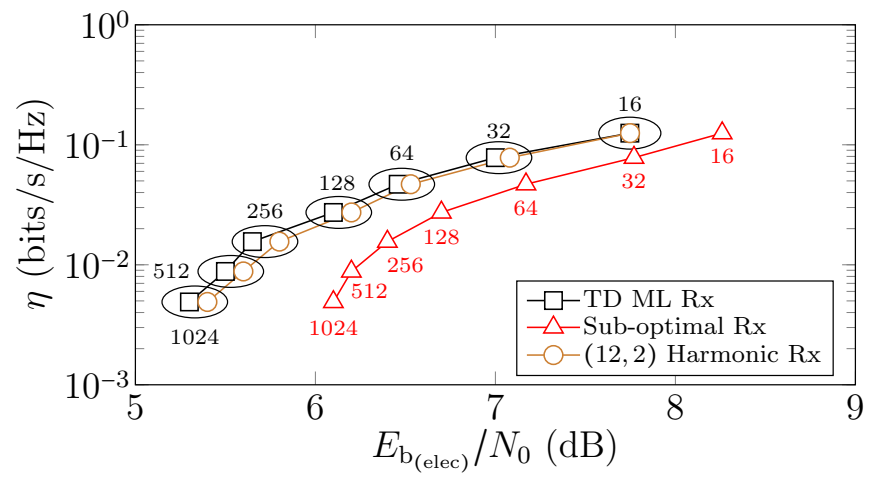

Fig. 5: Spectral efficiency as a function of the necessary $E_{\mathrm{b}_{(\text {elec }}} / N_{0}$ to reach a BER of $10^{-3}$ for the TD ML, sub-optimal and harmonic receivers, considering different $M$ in AWGN channel.

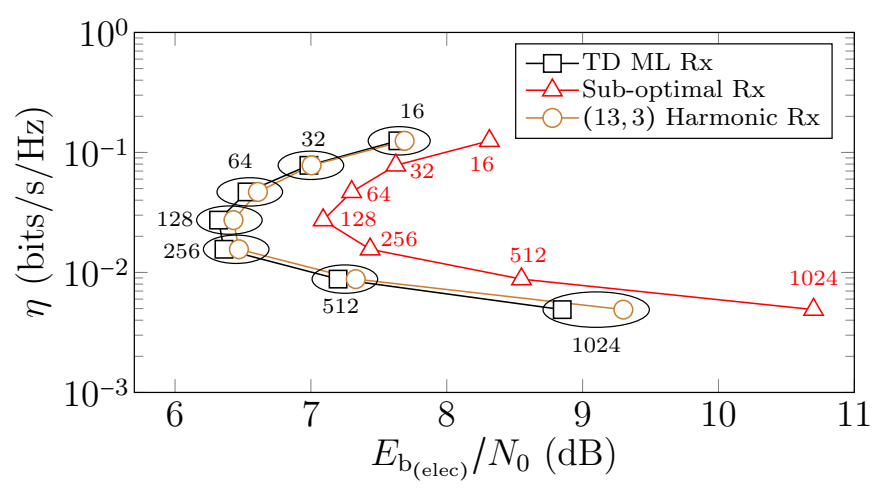

Fig. 6: Spectral efficiency as a function of the necessary $E_{\mathrm{b}_{(\mathrm{elec})}} / N_{0}$ to reach a BER of $10^{-3}$ for the TD ML, sub-optimal and harmonic receivers, considering different $M$ in time-dispersive channel.

on spectral features of U-FSK waveform, which define the minimum number of harmonics/taps needed for near-optimal performance. The simulation results show that the proposed receiver exhibits near-optimal performance in both AWGN and time-dispersive channels with significantly lowered complexity. The benefits of the proposed receiver should motivate further investigation into the above-mentioned challenges.

\section{REFERENCES}

[1] C.-W. Chen, W.-C. Wang, J.-T. Wu, H.-Y. Chen, K. Liang, L.-Y. Wei, Y. Hsu, C.-W. Hsu, C.-W. Chow, C.-H. Yeh, Y. Liu, H.-C. Hsieh, and Y.T. Chen. Visible light communications for the implementation of internetof-things. Optical Engineering, 55(6):060501, 2016.

[2] A. W. Azim, A. Rullier, Y. Le Guennec, L. Ros, and G. Maury. Energy efficient $M$-ary frequency-shift keying-based modulation techniques for visible light communication. IEEE Trans. Cogn. Commun. Netw., 5(4):1244-1256, 2019.

[3] A. W. Azim, Y. Le Guennec, and L. Ros. Hybrid frequency and phaseshift keying modulation for energy efficient optical wireless systems. IEEE Wire. Commun. Lett., 9(4):429-432, 2020.

[4] A. W. Azim, M. Chafii, Y. Le Guennec, and L. Ros. Spectral and energy efficient fast-OFDM with index modulation for optical wireless systems. IEEE Commun. Lett., 24(8):1771-1774, 2020.

[5] J. G. Proakis and M. Salehi. Digital communications. McGraw-Hill, 2008.

[6] J. Zhou, Y. Qiao, Z. Cai, and Y. Ji. Asymmetrically clipped optical fast OFDM based on discrete cosine transform for im/dd systems. J. Lightwav. Tech., 33(9):1920-1927, 2015.

[7] J. B. Carruthers and J. M. Kahn. Modeling of nondirected wireless infrared channels. IEEE Trans. Commun., 45(10):1260-1268, 1997.

[8] N. Fernando, Y. Hong, and E. Viterbo. Flip-OFDM for unipolar communication systems. IEEE Trans. Commun., 60(12):3726-3733, 2012. 